\title{
UV and melanoma: the TP53 link
}

\author{
Cell Research (2014) 24:1157-1158. doi:10.1038/cr.2014.95; published online 18 July 2014
}

Ultraviolet radiation (UVR) is a major risk factor for melanoma development, but it has been unclear exactly how UVR leads to melanomagenesis. In a recent publication in Nature, Viros et al. identify TP53/ $\operatorname{Trp} 53$ as a UVR-target gene in melanoma and show that UVR-induced TP53/Trp53 mutations accelerate BRAF(V600E)-driven melanomagenesis.

Melanoma is the deadliest skin cancer, and its incidence has relentlessly increased over recent decades. According to the American Cancer Society's estimates for melanoma in the United States for 2014, about 76100 new melanomas will be diagnosed and about 9710 people are expected to die from melanoma. It is well known that UVR is the major environmental factor contributing to melanomagenesis [1]. This suggests that there is at least a component of melanoma risk which may be preventable through UVR protective strategies - an issue of immense public health importance due to the availability of sunblocks and sun-safe behaviors. Importantly, while many studies have been conducted to elucidate the link between UVR and melanoma, the precise molecular mechanism(s) by which UVR triggers melanoma formation have remained incompletely understood.

Recently, a powerful UVR-induced skin inflammatory response has been shown to provoke metastasis of melanoma [2] and the presence of UV signature mutations has also been reported throughout the melanoma exome, including recurrent melanoma genes such as RAC1, PPP6C, and STK19 $[3,4]$. Previous mouse models for
UVR-induced melanoma revealed that UVR-induced inflammation promoted melanomagenesis in neonatal mice [5-7]. These studies underlined UVR's significant contribution to melanoma formation. In a recent study published in Nature, Viros et al. [8] address the role of UVR in previously established BRAF(V600E)-expressing melanocytes in vivo, and demonstrate that significantly accelerated melanoma formation often associated with mutations in TP53/ Trp53. To mimic both somatic mutation acquisition and mild sunburn in humans, BRAF(V600E) was expressed at physiological levels in adult mice which were subsequently exposed to repeated low doses of UVR. In addition, certain mice were partially covered with UVR-proof cloth or topically treated with SunSense Milk Sunscreen SPF50 $\left(2.2 \mathrm{mg} / \mathrm{cm}^{2}\right) 30$ min before UVR exposure, to assess the impact of these protective strategies.

UVR was seen to significantly accelerate melanoma formation in mice whose melanocytes express BRAF(V600E), but not in BRAF wild-type mice (which unlike BRAF(V600E)-expressing mice do not develop long latency melanomas independently of UVR). Application of UVR-proof cloth or sunscreen delayed the onset of UVR-driven melanoma and partially prevented acceleration of BRAF(V600E)-driven melanomagenesis by UVR, and sunscreen-protected UVR-exposed BRAF(V600E) mice developed a reduced number of melanomas compared with unprotected UVR-exposed BRAF(V600E) mice (Figure 1). More somatic single nucleotide variants and a significantly higher proportion of C-to- $\mathrm{T}$ transitions at the $3^{\prime}$ end of pyrimidine dimers were observed in UVR-exposed melanomas, providing direct evidence of UVR-induced DNA damage. In addition, Trp53 mutations (H39Y, S124F, R245C, R270C, C272G) were detected in UVR-exposed BRAF(V600E) mouse melanomas, indicating a direct role of UVR in the induction of Trp53 mutations in melanoma. The mutated corresponding residues (S127F/S124F, R248C/R245C, R273C/R270C, C275G/C272G) were also identified in TP53 mutations in human melanoma, suggesting that TP53 mutations are linked to evidence of UVR-induced DNA damage in human melanoma. These results are consistent with previous reports that $p 53$ deletion accelerates BRAF(V600E)-driven melanomagenesis both in mice [9] and in zebrafish [10], but demonstrate the ability of UVR to inflict UV signature mutations within the gene as has been widely observed in non-melanoma skin cancers and also in human melanomas.

This elegant study by Viros et al. clearly helps to establish key roles of UVR in melanomagenesis, and further validates the functional importance of TP53 as a UVR-targeted tumor suppressor gene in a fraction of melanomas. The study also raises several intriguing questions worthy of follow-up analysis. For example, through which mechanism(s) did sunscreen or sunshielding delay but not prevent UVR-induced melanoma? Induction of cutaneous inflammatory changes that are less anatomically restricted to UV irradiated fields, would seem to be an attractive mechanism. This may help to explain the known risk of melanoma in both sun-exposed and less-exposed skin of lightly pigmented people. It is also valuable to better un- 


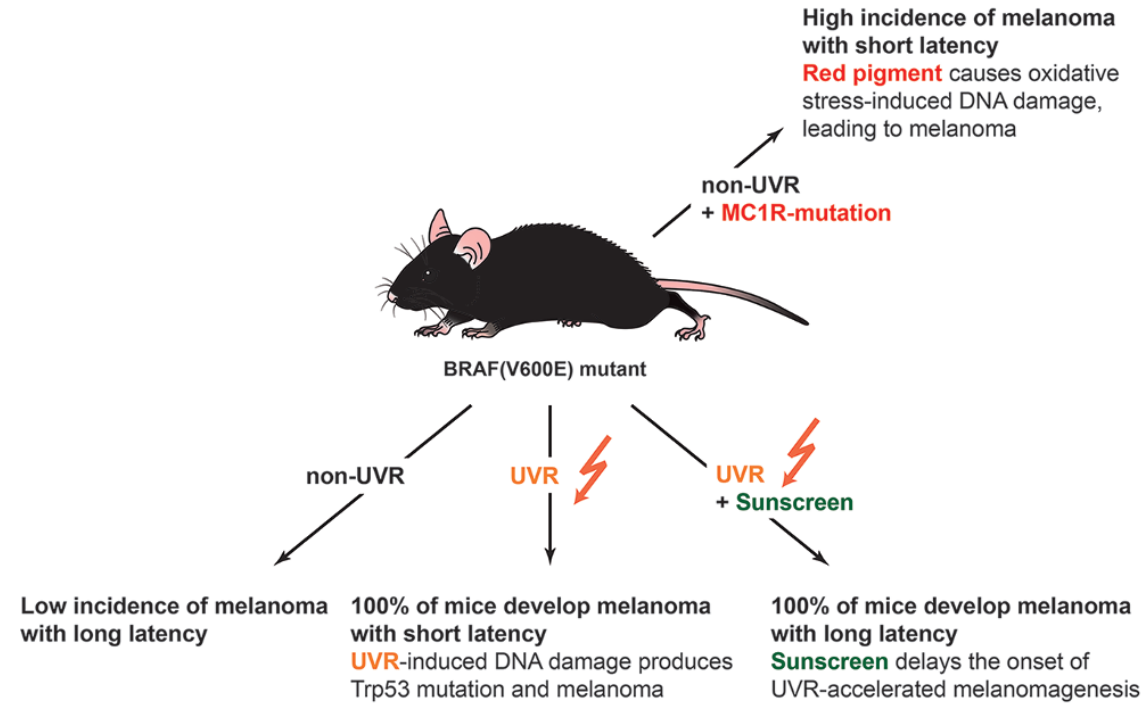

Figure 1 A diagram depicting feasible routes of $B R A F(V 600 E)$-driven melanomagenesis.

derstand the role of UVB vs UVA wavelengths in melanomagenesis. Mechanistically, these distinct regions of the UV spectrum inflict largely distinctive chemical alterations on the genome. Efforts to block UVA as well as UVB in commercial sunscreen products are currently being promoted by the US Food and Drug Administration, a welcome improvement to sun protection strategies. Still, the precise role(s) of UVA in melanomagenesis remain incompletely understood and may involve both cellautonomous and non-cell-autonomous targets. In addition to the acceleration of BRAF(V600E)-driven melanoma formation by UVR, red pigment (pheomelanin) has also been observed to accelerate BRAF(V600E)-driven melanomagenesis even in the absence of UVR [11]. Pheomelanin has been identified as an intrinsic risk factor for melanoma with the red pigment itself producing red pigment-induced BRAF(V600E)driven melanoma, and still remains as a major contributor to melanomagenesis. Therefore, along with UV shielding by sunscreens, further preventative strategies should be investigated to diminish UVR-independent melanoma risk mechanisms.

Viros et al. provide intriguing answers to several controversial questions regarding melanomagenesis: Does UVR really trigger melanoma? And can sunscreen actually prevent melanoma? The studies by Viros et al. provide experimental evidence for acceleration of BRAF(V600E)-driven melanoma by UVR-induced TP53/Trp53 mutation and demonstrate that sunscreen delayed but did not completely block
UVR-driven melanoma. The current study clearly shows that UVR boosts melanoma and sunscreens may provide partial UVR protection against melanoma - evidence which matches human epidemiologic data. Nevertheless, to protect the public from melanoma, Viros et al. advise that sunscreen should be utilized in combination with additional sun avoidance strategies. In addition, measures that may prevent UV-independent melanoma formation will require additional research and may also be needed in order to optimally battle the incidence of this life-threatening malignancy.

\section{Yeon Sook Choi $^{1}$, David E Fisher ${ }^{1}$}

${ }^{I}$ Cutaneous Biology Research Center, Department of Dermatology, Massachusetts General Hospital, Harvard Medical School, 55 Fruit Street, Boston, MA 02114, USA

Correspondence: David E Fisher

E-mail: dfisher3@partners.org

\section{References}

1 Garibyan L, Fisher DE. Curr Oncol Rep 2010; 12:319-326.

2 Bald T, Quast T, Landsberg J, et al. Nature 2014; 507:109-113.

3 Hodis E, Watson IR, Kryukov GV, et al. Cell 2012; 150:251-263.

4 Krauthammer M, Kong Y, Ha BH, et al. Nat Genet 2012; 44:1006-1014.

5 Noonan FP, Recio JA, Takayama H, et al. Nature 2001; 413:271-272.

6 Luo C, Sheng J, Hu MG, et al. Cancer Res 2013; 73:4337-4348.

7 Zaidi MR, Davis S, Noonan FP, et al. Nature 2011; 469:548-553.

8 Viros A, Sanchez-Laorden B, Pedersen M, et al. Nature 2014; 511:478-482.

9 Goel VK, Ibrahim N, Jiang G, et al. Oncogene 2009; 28:2289-2298.

10 Patton EE, Widlund HR, Kutok JL, et al. Curr Biol 2005; 15:249-254.

11 Mitra D, Luo X, Morgan A, et al. Nature 2012; 491:449-453. 